\title{
Immune cell and cytokine patterns in children with type 1 diabetes mellitus undergoing a remission phase: A longitudinal study
}

\author{
Ana Laura Fitas $^{1}$ [ | Catarina Martins ${ }^{2}$ | Luís Miguel Borrego ${ }^{2}$ | Lurdes Lopes ${ }^{1}$ | \\ Anne Jörns $^{3}$ | Sigurd Lenzen ${ }^{3,4}$ | Catarina Limbert ${ }^{1}$
}

\author{
${ }^{1}$ Paediatric Endocrinology Unit, Hospital de \\ Dona Estefânia, Centro Hospitalar de Lisboa \\ Central, Lisbon, Portugal \\ ${ }^{2}$ Chronic Diseases Research Center CEDOC- \\ NOVA Medical School, Lisbon, Portugal \\ ${ }^{3}$ Institute of Clinical Biochemistry, Hannover \\ Medical School, Hannover, Germany \\ ${ }^{4}$ Institute of Experimental Diabetes Research, \\ Hannover Medical School, Hannover, \\ Germany \\ Correspondence \\ Dr Ana Laura Fitas, Paediatric Endocrinology \\ Unit, Hospital de Dona Estefânia, Centro \\ Hospitalar de Lisboa Central; Rua Jacinta \\ Marto, Lisbon 1169-045, Portugal. \\ Email: ana.fitas@chlc.min-saude.pt \\ Funding information \\ Sanofi
}

\begin{abstract}
Objective: Type 1 diabetes (T1D) develops in distinct stages, before and after disease onset. Whether the natural course translates into different immunologic patterns is still uncertain. This study aimed at identifying peripheral immune patterns at key time-points, in T1D children undergoing remission phase.

Methods: Children with new-onset T1D and healthy age and gender-matched controls were recruited at a pediatric hospital. Peripheral blood samples were evaluated by flow cytometry at 3 longitudinal time-points: onset (T1), remission phase (T2) and established disease (T3). Cytokine levels were quantified by multiplex assay. Fasting C-peptide, HbA1c, and 25OHD were also measured.

Results: T1D children ( $n=28 ; 10.0 \pm 2.6$ years) showed significant differences from controls in circulating neutrophils, T helper (Th)17 and natural killer (NK) cells, with relevant variations during disease progression. At onset, neutrophils, NK, Th17 and T cytotoxic (Tc)17 cells were decreased. As disease progressed, neutrophil counts recovered whereas NK counts remained low. Th17 and Tc17 cells behavior followed the neutrophil variation pattern. B-cells were lowest in the remission phase and regulatory T-cells significantly declined after remission. Two cytokine response profiles were identified. Low cytokine-responders showed higher circulating fasting C-peptide levels at onset and longer remission periods. C-peptide inversely correlated with pro-inflammatory and cytotoxic cells.

Conclusions: Our data suggest an association between immune cells, cytokine patterns and metabolic counterparts. The dynamic changes of circulating immune cells during disease progression involve key innate and acquired immune cell types. This longitudinal picture of T1D progression may enable disease staging and patient stratification, essential for individualized treatment.
\end{abstract}

\section{KEYWORDS}

c-peptide, cytokines, diabetes mellitus, type 1, lymphocytes, neutrophils

\section{1 | INTRODUCTION}

Type 1 diabetes (T1D) is a metabolic disorder in which the interaction between genetic susceptibility, environmental factors and immunologic mechanisms leads to a selective destruction of pancreatic insulin-producing beta-cells. ${ }^{1}$ This chronic and life-debilitating disease affects a growing number of young children, ${ }^{2}$ yet its etiology and pathogenesis are still unresolved. A T effector/T regulatory (Treg) cell imbalance has been considered, allowing the expansion of cytotoxic $\mathrm{CD}^{+} \mathrm{T}-^{3}$ and B-cells, ${ }^{4}$ as observed in animal models, along with autoantibody production. T1D is also related to MHC-gene polymorphisms ${ }^{5}$ implying an involvement of the adaptive immune system. 
Nevertheless, increasing evidence suggests that innate immune cells and non-specific inflammation also play a critical role in the pathogenesis of T1D, as shown by studies in pancreatic insulitis and peripancreatic ganglia. $^{6,7}$

Along the natural history of T1D, periods of immunologic tolerance and autoimmune activity occur, probably as a consequence of antigen determinants spreading, beta-cell proliferation or cytotoxic T-cell action. ${ }^{8}$

It is well recognized that in $40 \%$ to $71 \%$ of children, ${ }^{9,10}$ T1D develops in distinct phases, corresponding to different metabolic and possibly immunological stages. ${ }^{8}$ After onset, a remission phase or "honeymoon" may occur, with a partial and transitory restoration of endogenous insulin production. This natural phenomenon has been attributed to lower metabolic glucotoxicity, due to action of exogenous insulin and glycaemic improvement. Several authors have hypothesized that the "honeymoon" is a phase of immunotolerance in a relapse-remitting disease..$^{8,11}$

Studies approaching peripheral blood (PB) and target organ are crucial to identify biomarkers as predictors for early and individualized intervention. Moreover, the dynamics of disease course in humans requires a thorough investigation to identify an optimal therapeutic window for induction of beta-cell regeneration and restoration of immunotolerance.

Current knowledge of T1D pathogenesis relies mostly on animal models, which do not fully mirror the human T1D pathology, as recently documented in a direct comparative study. ${ }^{12}$ On the other hand, the risks of presently available techniques to access the pancreas in vivo restrain organ studies in humans, and consequently human data on disease progression are rare. ${ }^{13,14}$

Circulating immune cells in T1D patients may reflect the events occurring in the pancreas ${ }^{15}$ and may predict the occurrence of the remission phase, ${ }^{16}$ but prospective longitudinal data are limited. One study in children with T1D suggested that specific immunological signatures are related to disease severity ${ }^{17}$ and functional changes in circulating Treg have been reported at different disease stages. $^{18}$

Thus, we hypothesize that changes in the profile of PB immune cell subsets and cytokines may reflect the natural course of T1D in humans. This study aimed to identify peripheral immune patterns in T1D children during disease progression, and to determine whether a link between the inflammatory status and metabolic severity exists. The longitudinal picture of T1D progression may enable disease staging and patient stratification, which will allow for individualized treatment.

\section{2 | METHODS}

\section{1 | Study population}

Children with new-onset T1D were recruited at admission for 18 months in a central pediatric hospital. Inclusion criteria were: age 5 to 17 years old, and <14 days after diagnosis. T1D was defined according to the ADA criteria, ${ }^{19}$ with at least one positive autoantibody (anti-insulin; Insulinoma-Associated-2; antiglutamic acid decarboxylase). Children with other autoimmune diseases, allergy or acute infections were excluded. Controls (age and gender-matched) were selected from the pediatric endocrinology outpatient clinic, where they were evaluated due to variants of normal growth and/or puberty. Initially, 42 patients were recruited, of which 28 completed the study (Figure 1).

\section{2 | Study design}

Blood samples were collected at 3 distinct time-points throughout individual disease development. At disease onset (time-point 1: T1), samples were collected between 1 and 14 days after diagnosis. At partial remission (time-point 2: T2) and at "established disease" (timepoint 3: T3), samples were collected according to the value of Insulin dose adjusted to $\mathrm{HbA}_{1 \mathrm{c}}\left(\mathrm{IDAA}_{1 \mathrm{c}}\right)$, used as a surrogate measure of endogenous insulin production. IDAA $\mathrm{ID}_{1 \mathrm{C}}=\mathrm{HbA}_{1 \mathrm{C}}(\%)+(4 \times$ insulin dose [units per kilogram per 24 hours]). ${ }^{20} \mathrm{~T} 2$ was collected once the patient entered partial remission phase $\left(\right.$ IDAA $\left._{1 \mathrm{C}} \leq 9\right)$ and T3 was collected once patients entered established disease phase, that is, $\mathrm{IDAA}_{1 \mathrm{C}}>9$.

Control samples were collected simultaneously to T1. Longitudinal data collection occurred in a 2-year time-period. The number of cases admitted to the hospital during the study period determined the initial sample size. For study evaluation, only patients with successful sample collection in all time-points were considered (Figure 1).

The study was approved by the hospital's ethics committee and conducted in accordance with the Declaration of Helsinki. Informed consent forms were signed.

\section{3 | Sample collection}

Blood samples for immunophenotyping and cell stimulation were collected in EDTA and heparin tubes, respectively, and processed within 24 hours. For cytokine quantification, sera were obtained by centrifugation after clot retraction, separated in aliquots and stored at $-20^{\circ} \mathrm{C}$ until analysis.

\section{4 | Flow cytometry}

Multicolor flow cytometry was used for characterization of immune cell subsets, using a BD FACS Calibur (BD Biosciences, San Jose, California) equipped with 2 lasers (488 nm air-cooled argon-ion laser, and $635 \mathrm{~nm}$ red diode laser). For characterization of leukocyte and lymphocyte subpopulations the BD Multitest IMK Kit with Trucount absolute counting tubes (BD Biosciences) was used, according to manufacturer's instructions.

For Treg phenotyping, the panel of mAbs included anti-CD3FITC (clone SK7, BD Biosciences), anti-CD25 PE (clone BC96, Biolegend, California), anti-CD4 PerCPCy5.5 (clone SK3, Biolegend) and anti-CD127 AlexaFluor647 (clone A019D5, Biolegend) (Additional information in Figure S1, Supporting Information). ${ }^{21,22}$ 
FIGURE 1 Study design.

T1D, type 1 diabetes;

T1, time-point 1 (new-onset disease);

T2, time-point 2 (remission phase);

T3, time-point 3 (established disease);

$I_{1 D A} A_{1 C}$, insulin dose adjusted $\mathrm{HbA}_{1 \mathrm{C}}$
Diagnosis $-\mathrm{T} 1: 4 \pm 2$ days
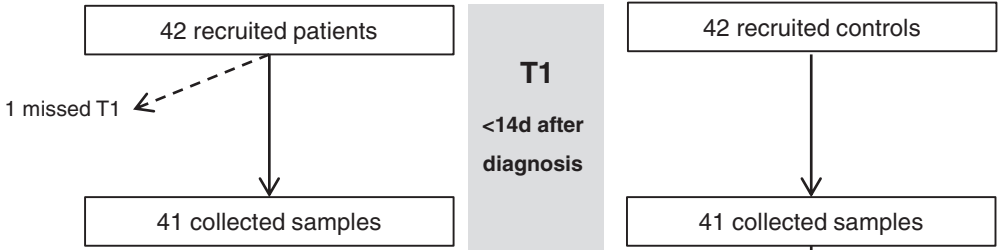

T1 - T2: $107 \pm 46$ days

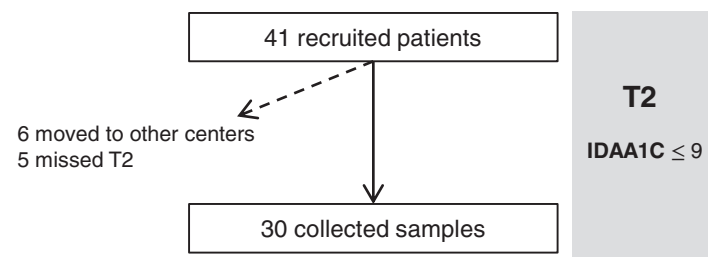

T2 - T3: $279 \pm 118$ days

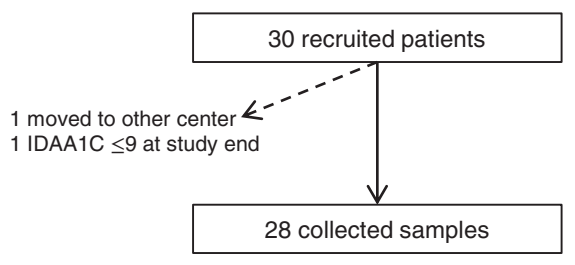

T3

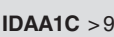

T1 - T3: $398 \pm 104$ days

\subsection{Cell stimulation for intracellular cytokine} evaluation

The expression of IFN- $\gamma$ and IL17 was used to assess Th1 and Th17 cells. For this, heparinized whole blood samples were stimulated with PMA (50 ng/mL; Sigma Aldrich, St. Louis, Missouri) and calcium ionophore $\left(1 \mu \mathrm{g} / \mathrm{mL}\right.$, Sigma Aldrich) for 5 hours at $37^{\circ} \mathrm{C}$ in a $5 \% \mathrm{CO}_{2}$ atmosphere, in the presence of Brefeldin A $(1.0 \mu \mathrm{g} / \mathrm{mL}$, BD Pharmingen, San Jose, California). For control purposes, unstimulated cells were incubated in parallel. The protocol was performed according to the Cytofix-Cytoperm kit (BD Pharmingen) instructions (Additional information in Figure S2).

\subsection{Serum cytokines quantification by Luminex technology}

Serum cytokines (IL-1 $\beta$, IL-2, IL-4, IL-6, IL-10, IL-13, IL17-A, IFN- $\gamma$, MCP-1 and TNF- $\alpha$ ) were accessed by a multiplex strategy. A 10-plex Bioplex Human Cytokine Assay kit (Bio-Rad Laboratories, Inc., Hercules, California) was used for the quantification, according to manufacturer recommendations.

\section{7 | Indicators of metabolic status}

Glycated hemoglobin $\mathrm{HbA}_{1 \mathrm{C}}$ was determined by chromatography (Variance 2-Biorad, Hercules, California) and fasting C-peptide and 25-OHD were determined by chemiluminescence (Immulite 2000, Siemens, Germany).

\section{8 | Statistical analysis}

GraphPad Prism software v.6.01 (GraphPad Software, La Jolla, California) was applied. Categorical variables were analyzed by Fisher's exact test or $\chi^{2}$. Normality of distributions was assessed by D'Agostino \& Pearson test. Data normally distributed were presented as mean $\pm S D$, otherwise as median and interquartile range. Paired groups were analyzed with paired Student's $t$ test or Wilcoxon test. Unpaired Student's $t$ or Mann-Whitney tests were used to compare each 2 independent groups. $P<.05$ was considered significant.

\section{3 | RESULTS}

\subsection{Characteristics of the study population}

Table 1 summarizes demographic and metabolic data from T1D patients and controls. The T1D group comprised 28 children (mean age: $10.0 \pm 2.6$ years, $46 \%$ males). At disease onset (T1), samples were collected $4 \pm 2$ days after diagnosis. At partial remission (T2), average sample collection time was $107 \pm 46$ days after diagnosis, and at established disease (T3) $398 \pm 104$ days after diagnosis. Remission phase (T2-T3) duration was on average $279 \pm 118$ days (Figure 1). No relevant seasonal clustering was identified.

\subsection{Neutrophils and NK-cells were decreased at T1D onset with regain of neutrophils but not of NK- cells}

Compared to controls, both neutrophils and NK-cell counts were significantly decreased in TD1 children at disease onset $(P=.0439$; 
TABLE 1 Characteristics of the study population

\begin{tabular}{lllllllll} 
& $\begin{array}{l}\text { Age, years } \\
\text { (mean, SD) }\end{array}$ & $\begin{array}{l}\text { Sex } \\
\text { (M/F) }\end{array}$ & $\begin{array}{l}\text { Onset BMI, kg/m² } \\
\text { (mean, SD) }\end{array}$ & $\begin{array}{l}\text { Positive } \\
\text { AIA }\end{array}$ & $\begin{array}{l}\text { Positive } \\
\text { IA2 }\end{array}$ & $\begin{array}{l}\text { Positive } \\
\text { GAD }\end{array}$ & $\begin{array}{l}\text { Onset HbA } \\
\text { (mean, SD) }\end{array}$ & $\begin{array}{l}\text { Onset fasting C-peptide, ng/mL } \\
\text { (median, IQR) }\end{array}$ \\
\hline Patients $(n=28)$ & $10(3)$ & $14 / 14$ & $16.8(3.0)$ & $24 / 28$ & $9 / 28$ & $10 / 28$ & $12.7(2.4)$ & $0.3(0.2 ; 0.6)$ \\
Controls $(n=28)$ & $10(3)$ & $14 / 14$ & - & - & - & - & - & -
\end{tabular}

Abbreviations: AIA, anti-insulin autoantibody; F, female; GAD, antiglutamic acid decarboxylase autoantibody; IA2, insulinoma-associated-2 autoantibody; $\mathrm{IQR}$, interquartile range; $M$, male; $S D$, standard deviation.

$P=.0115$, respectively) (Figure 2B,D). The frequency of NK-cells was also significantly lower $(P=.0012)$ (Figure $2 C)$. Upon disease progression, neutrophils increased, both in absolute counts (T1-T2 $P=.1375$; T1-T3 $P=.0082 ;$ T2-T3 $P=.0112)$ and frequency (T1-T2 $P=.0564$; T1-T3 $P=.0005 ;$ T2-T3 $P=.0089)$. This neutrophil recovery was most pronounced at the final stage, reaching the control group values (Figure 2A,B). Similarly, NK-cells significantly increased during disease progression (T1vsT2 $P=.0139 ;$ T1vsT3 $P=.0188 ;$ T2vsT3 $P=$.9670). However, absolute counts and percentages remained diminished at T3 (respectively, $P=.0307 ; P=.0789$ ) compared to controls (Figure 2C,D).

\section{3 | B-cells reached their lowest level during the remission phase}

B-cells significantly decreased from $\mathrm{T} 1$ to $\mathrm{T} 2$, without further changes, both in absolute number (T1-T2 $P=.0002 ;$ T1-T3 $P=.0006)$ and frequency (T1-T2 $P=.0003 ;$ T1-T3 $P=.0075)$ (Figure 2E,F). No correlation was identified for B-cells, considering metabolic parameters (C-peptide, $\mathrm{HbA} 1 \mathrm{c}$, and vitamin $\mathrm{D}$ ) and duration of disease stages.

\subsection{The regain of pro-inflammatory Th1 and Th17 cells after T1D onset was accompanied by a decline of regulatory $\mathrm{T}$-cells after remission}

Compared to control values, Th1 and Tc1 cell subsets did not show significant differences in T1D children at any time-point. However, a significant increase was detected during the disease course (Th1\% T1-T2 $P=.0041$, T1-T3 $P=.0014 ;$ Tc1\% T1-T2 $P=.0032, \mathrm{~T} 1-\mathrm{T} 3$ $P=.0004)$. The rise of these pro-inflammatory cells occurred mainly in stage T2 (Figure 3A,B). Differently, Th17 and Tc17 cell populations were significantly decreased, in T1D children compared to controls, already at disease onset (Th17\% $P=.0047$, Th17 cells $/ \mu \mathrm{L} P=.0155$; Tc17\% $P=.0003$, Tc17 cells $/ \mu \mathrm{L} P=.0017)$. Although there was a significant increase during disease progression (Th17\% T1-T2 $P=.0322$; T1-T3 $P=.0207 ;$ Tc17\% T1-T3 $P=.0218$ ), both populations remained below control levels (Figure 3C,D). Treg frequency and numbers were not significantly different from controls at any of the 3 time-points. However, Treg significantly decreased in the established disease phase (Treg\% T2-T3 $P=.0016$; Treg cells/ $\mu \mathrm{L}$ T1-T3 $P=.0028$; T2-T3 $P=.0497$ ) (Figure 3E,F). Addressing the ratios, Th17/Treg was significantly lower at disease onset, compared to controls $(P=.0117)$. Th1/Treg was not different at any time-point. Along the disease course, both ratios significantly increased (Th1/Treg T1-T3 $P=$.0036; Th17/Treg T1-T3 $P=.0118$ ).

\section{5 | C-peptide at T1D onset was related to the immune cell pattern and duration of the remission phase}

At disease onset, C-peptide inversely correlated with absolute counts of pro-inflammatory and cytotoxic cells, namely T-cells $(r=-.395$; $P=.042)$ (Figure 4A), NK-cells $(r=-0.486 ; P=.010)$ (Figure 4B), Tc1 cells $(r=-0.479 ; P=.011$ ) (Figure $4 \mathrm{C}$ ) and total IFN- $\gamma$-secreting CD8 T-cells $(r=-0.481 ; P=.011)$. A positive correlation between $\mathrm{C}$-peptide at disease onset and remission duration was also identified $(r=0.381 ; P=.050)$. Children with lower $C$-peptide at onset had shorter remission phases.

\subsection{Limited impact of vitamin D status at T1D onset}

To address the effect of seasonality, we compared T1D patients with disease onset from November to April with patients diagnosed from May to October. Our results revealed no significant differences regarding vitamin D levels and immune cells and cytokines in the 2 groups. Also, at T1D onset, vitamin D levels did not correlate with any of the immune parameters evaluated (cell subsets and cytokines).

\section{7 | Serum cytokines at disease onset were related to $C$-peptide levels and duration of the remission phase}

Regarding cytokine concentrations, no differences were found for any studied cytokines between T1D children (T1, T2, or T3) and controls; neither significant variation along disease progression was observed.

However, we identified 2 cytokine patterns within our patients: low responders (LR), with no detectable anti-inflammatory cytokines (IL-4, IL-10, IL-13) and no pro-inflammatory TNF- $\alpha$; high responders (HR) with at least one of these cytokines detected (IL-4, IL-10, IL-13, or TNF- $\alpha$ ). Consistently, the HR group presented higher levels of other cytokines such as IL2 ( $P=.038)$, IL-6 $(P=.002)$, IFN- $\gamma(P<.001)$ and MCP-1 $(P=.004)$ at onset; IL2 ( $P=.014)$, IL-17A $(P=.022)$ and IFN- $\gamma$ $(P=.046)$ at partial remission and IL1- $\beta(P=.019)$, IL2 ( $P=.003)$, IL-6 $(P=.004)$, IL-17A $(P=.035)$, IFN- $\gamma(P=.002)$ and MCP-1 $(P=.009)$ after disease establishment.

LR had both higher circulating fasting $C$-peptide levels at disease onset $(P=.008)$ and longer remission periods $(P=.005)$ compared to HR (Figure 5A,B). There were no differences between $L R$ and HR regarding immune cell subsets. No correlation between cytokine levels and age was identified. 

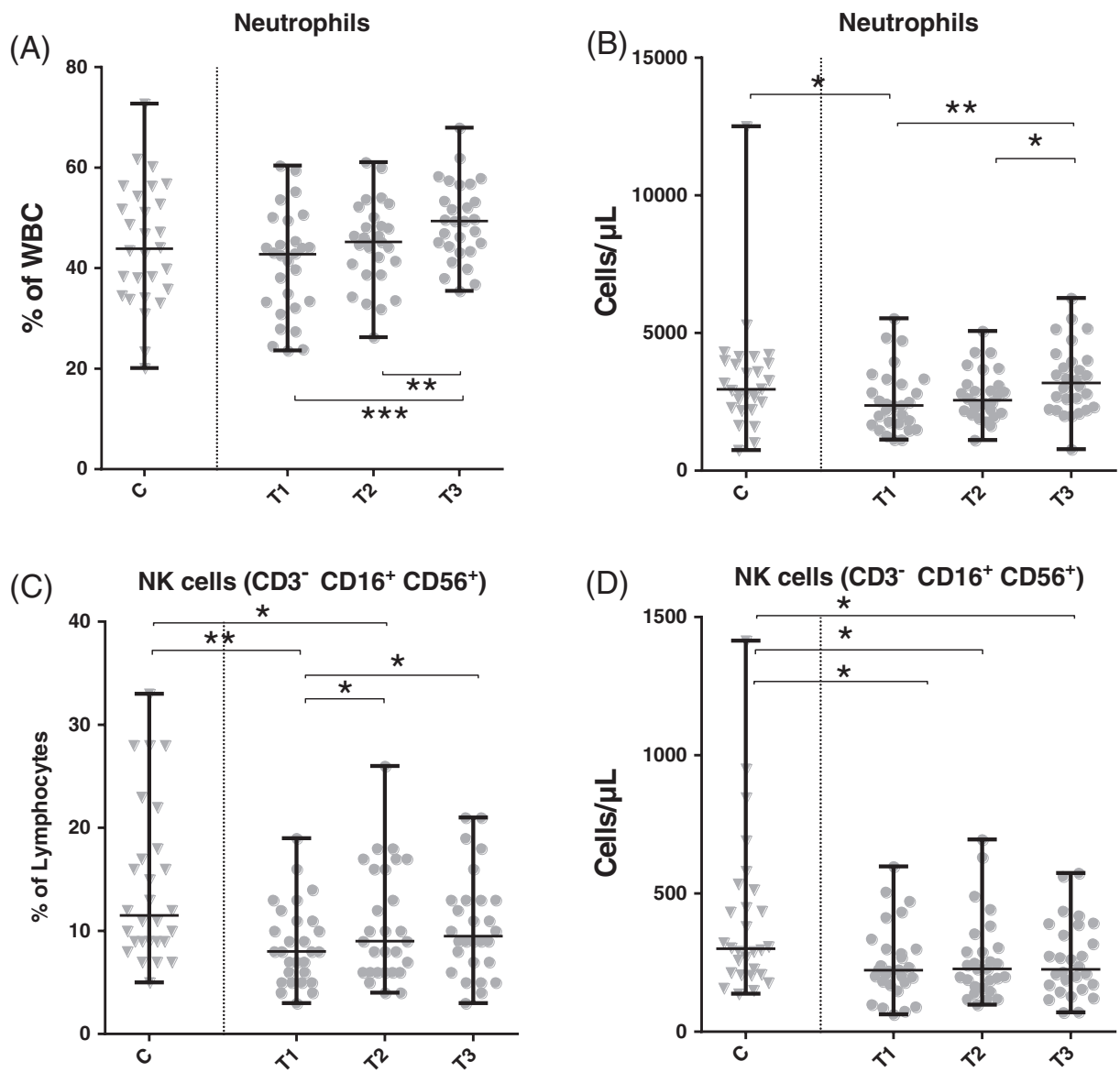

FIGURE 2 Neutrophils, NK and B-cells

(E)

(F)

B cells (CD19 $)$ peripheral immune pattern from T1D onset to established disease. Percentages and absolute counts of peripheral neutrophils (A, B), NK (C, D) and B-cells (E, F) from T1D children and healthy controls. PB collection at disease onset (T1), partial remission (T2) and disease establishment (T3). $* P<.05$, $* * P<.01, * * * P<.001$ by MannWhitney test (patients vs controls) or Wilcoxon matched-pairs signed rank test (comparison within patients in different time-points)
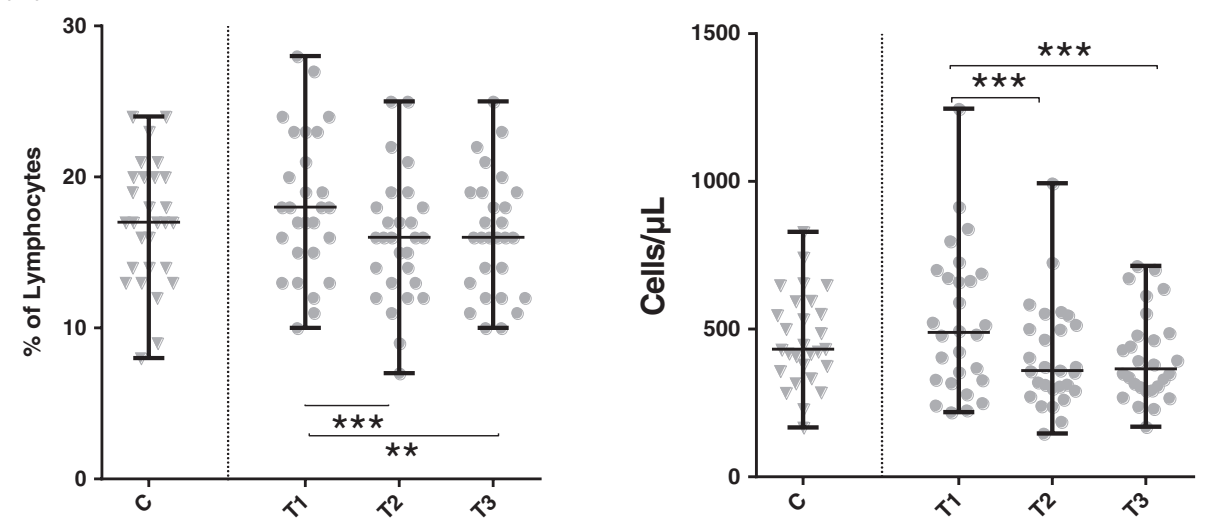

\section{4 | DISCUSSION}

Various models of T1D disease progression have been published, based mostly on animal studies. ${ }^{12}$ To the best of our knowledge, this is the first study which prospectively monitors immune cell subsets and cytokines in T1D children from disease onset throughout the remission phase up to disease final establishment.

T1D children differed significantly from healthy controls in circulating neutrophils, Th17 and NK-cells, with relevant variations at onset, partial remission and disease establishment. In addition, B-cells, Treg and IFN- $\gamma$ producing T-cells significantly varied during disease progression. The natural history of T1D seems to be modulated by immune parameters, as they correlate with residual insulin production. Considering the cytokine environment, 2 profiles were identified in T1D patients, low and high responders, with significantly different basal C-peptide levels at disease onset as well as distinct periods of remission.

The neutrophil reduction at disease onset has previously been described in humans with T1D but was not evident in T2D patients, suggesting that such a decrease is not due to impaired glycaemic control. $^{23}$ The reason for decreased neutrophil counts is most likely tissue sequestration. Accordingly, data on T1D pancreas morphology have shown neutrophil infiltration in exocrine pancreas at disease onset. $^{23,24}$ Recruitment of these innate immune cells to the pancreas by the pro-inflammatory IL-17 environment is one of the proposed mechanisms by which neutrophils participate in T1D. ${ }^{7}$ Furthermore, 
(A)

Th1 (IFN- $\left.{ }^{+}\right)$

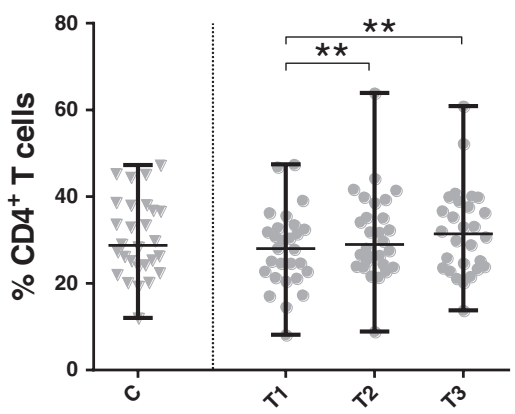

(C)

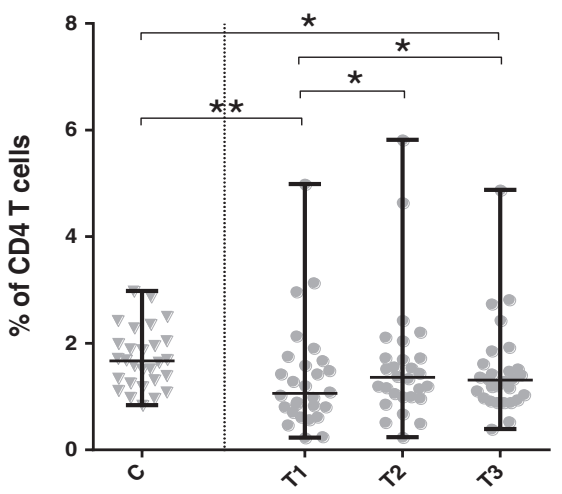

(E)

$$
\text { Treg CD4 }{ }^{+} \text {CD25 }{ }^{+} \text {CD127 } 7^{-/ d i m}
$$

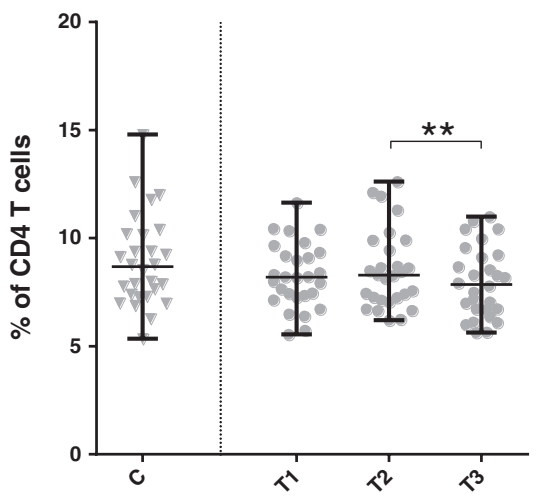

(B)

Tc1 (IFN- $\left.\gamma^{+}\right)$

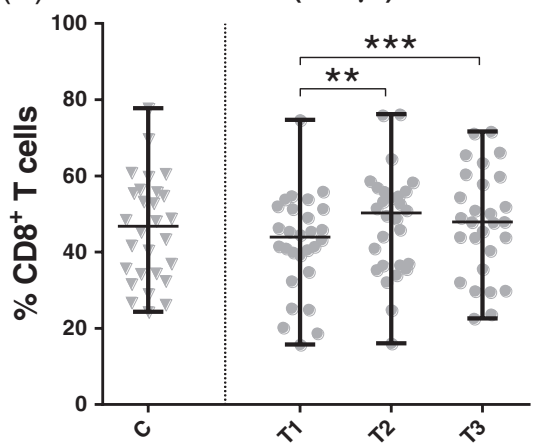

(D)

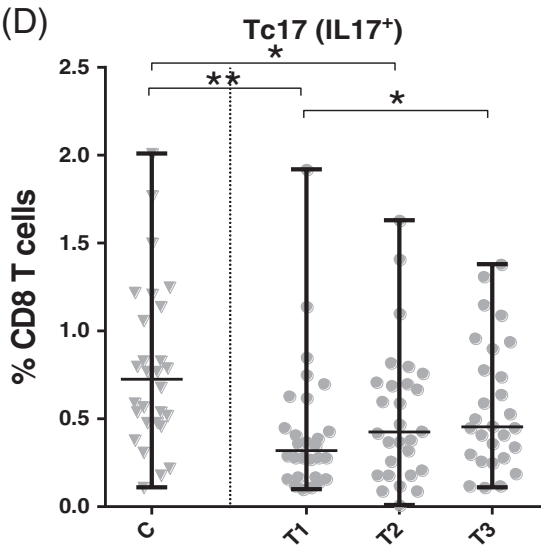

(F)

Treg CD4 ${ }^{+} \mathrm{CD} 25^{+} \mathrm{CD} 127^{-/ \mathrm{dim}}$

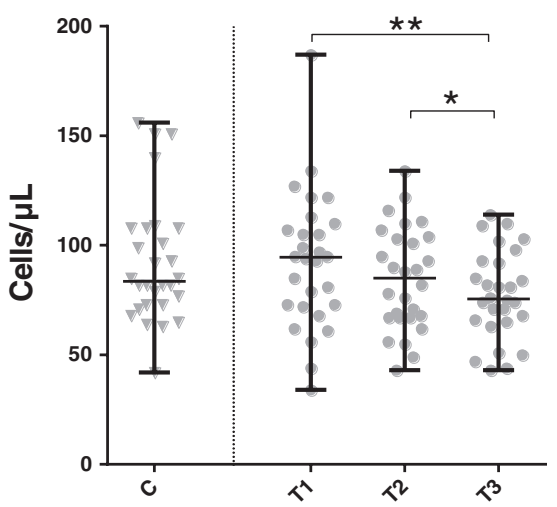

FIGURE 3 T-cell differentiation subsets from T1D onset to established disease. Peripheral pattern of Th1 (A), Tc1 (B), Th17 (C), Tc17 (D) and Treg (E, F) from T1D children and healthy controls. PB collection at disease onset (T1), partial remission (T2) and disease establishment (T3). $* P<.05$, $* * P<.01$, *** $P<.001$ by Mann-Whitney test (patients vs controls) or Wilcoxon matched-pairs signed rank test (comparison within patients in different time-points)
IL17-producing cells are involved in both neutrophil recruitment and activation at inflammatory sites. ${ }^{25}$ Similar to other autoimmune diseases, significant migration of Th17 cells and neutrophils to the target organ can play a major role in early disease stages. ${ }^{26}$

Evidence for Th17 cells in the pancreas ${ }^{27}$ and pancreatic lymph nodes, ${ }^{28}$ as well as the presence of neutrophils in pancreatic tissues of T1D donors, but not of healthy donors, ${ }^{23}$ supports the recruitment of neutrophils into inflammatory areas, leading to pancreatic tissue damage. ${ }^{7}$ Circulating neutrophil reduction went along with the early phase of active, ongoing and specific autoimmune pancreatic betacell destruction. ${ }^{7}$ In line with this hypothesis, we observed that neutrophils positively correlated with the duration of remission phase (unpublished results).

In our T1D population, neutrophils started to recover at remission, significantly increasing at established disease. Differently, prolonged mild neutropenia has been described, with later resolution,
5 years after diagnosis. ${ }^{23}$ An explanation for these different results might be the relapsing-remitting character of T1D disease course. We speculate that a regain of neutrophils during remission phase results not only from a reduction of migratory events toward the pancreas, but also from increased medullary neutrophil differentiation, promoted by Th17 immunity. ${ }^{29}$ A similar pattern was evident in our study for both neutrophils and IL17-producing cells in T1D patients, reinforcing that the Th17/neutrophil pathway is primarily involved in the immunopathology of T1D. ${ }^{7}$ Reports on circulating levels of IL-17producing cells in T1D are controversial, showing either increased ${ }^{30,31}$ or unchanged levels compared to controls. ${ }^{32}$ It may be argued that lower numbers of peripheral IL-17-producing cells reflect increased recruitment to the target organ and draining lymph nodes that outweighs numbers of circulating cells. ${ }^{28}$ In addition, the synergism between Th17 and Th1 cells is generally accepted in T1D pathogenesis. ${ }^{27}$ Accordingly, we have identified double positive IL-17 ${ }^{+} \mathrm{IFN}-\gamma^{+}$ 


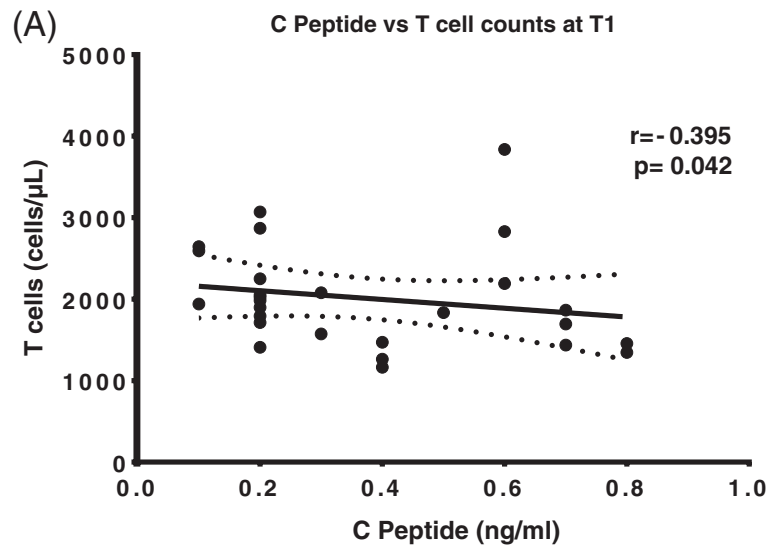

(B) C Peptide vs $\mathrm{N} K$ cell counts at $\mathrm{T} 1$

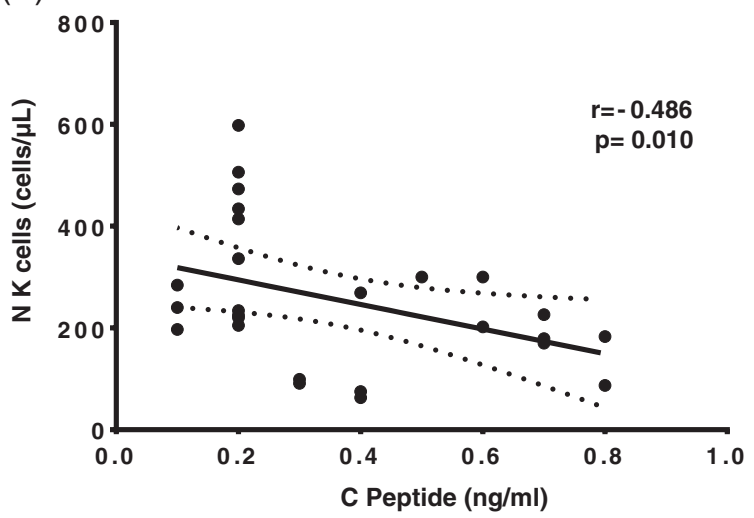

(C) C Peptide vs Tc1 cell counts at T1

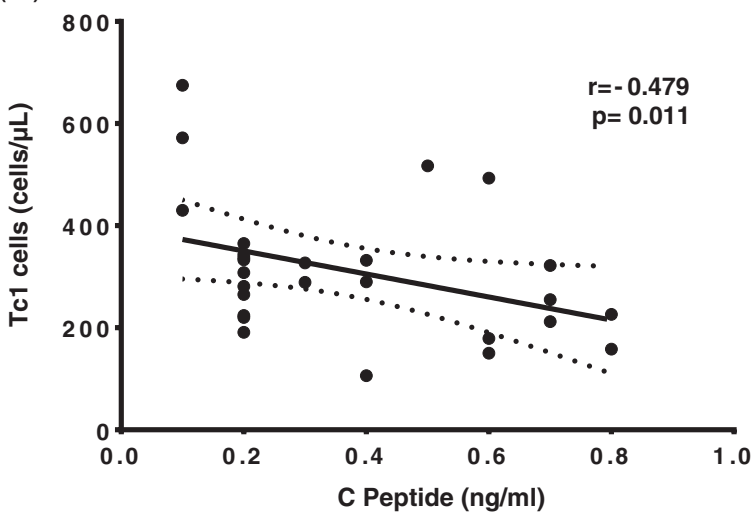

FIGURE 4 Correlation between immune cell populations and fasting C-peptide at T1D onset. T-cell (A), NK (B) and Tc1 (C) absolute counts are shown in correlation to fasting $C$-peptide. $P<.05$ by Spearman's rank order correlation

secreting T-cells in T1D and controls (unpublished observation), confirming earlier observations. ${ }^{31}$

In accordance with previous studies, Treg counts did not differ between patients and controls. ${ }^{18,33}$ However, a Treg depletion has been described in new-onset ${ }^{34}$ and long-standing T1D. ${ }^{34,35}$ Differences in phenotyping strategies used for Treg identification may explain discrepancies between studies. ${ }^{30}$ On the other hand, the significant decrease in Tregs at T3 may reflect a quantitative impairment resulting from long-term activation of these cells during the chronic autoimmune inflammatory process. ${ }^{36}$ The increase in Th17/Treg ratio observed during the disease course in our T1D children has been described before. ${ }^{37}$ This dynamics highlights again the role of Th17

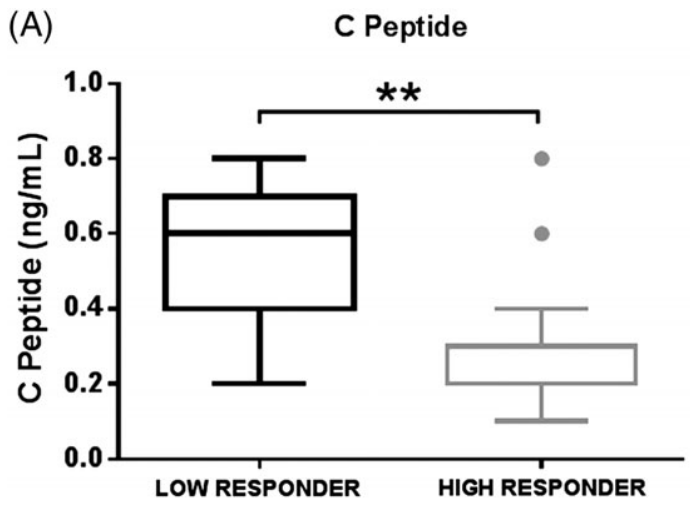

(B) Duration of remission phase

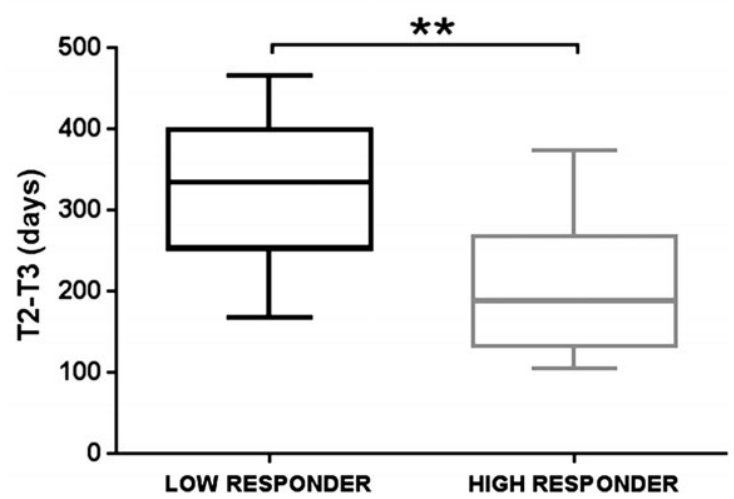

FIGURE 5 Fasting C-peptide levels and remission phase duration in T1D children with low and high-cytokine responses. Fasting C-peptide (A) and duration of remission phase (B) were compared between patients in 2 categories of cytokine response: low responders (LR) and high responders (HR). **P $<.01$ by MannWhitney test

immunity in T1D, ${ }^{38,39}$ sustaining the idea that the classic Th1/Th2 paradigm is not sufficient to explain the immunopathogenic events that lead to autoimmune diabetes.

Though NK frequencies varied in different studies reduced NK counts have been previously described in T1D children. ${ }^{40}$ Whether NKs are disease-controlling or disease-promoting cells in T1D is still unclear. ${ }^{41}$ However, the fact that T1D children with higher levels of NK-cells have shorter remission phases (unpublished observation) suggests a pathogenic pancreatic involvement of NK-cells in T1D.

Increased circulating cytotoxic cells, namely NK-cells or Tc1 cells, and a higher cytokine response in those children with lower Cpeptide levels at disease onset reflects a close relation between immune subsets and metabolic counterparts in T1D children. Therefore, a link between more severe disease patterns and inflammationprone environment is likely to exist.

Cytokines are known to be involved in autoimmune destruction of beta-cells. ${ }^{42,43}$ Other groups have attempted to use single cytokines as disease progression markers, but no correlations were found during the first year of disease. ${ }^{16}$ Pro-inflammatory cytokines in our T1D patients were comparable to age-matched healthy controls, similar to recent data on circulating IL-17 in T1D children and controls under 15 years of age ${ }^{44}$ as well as to cytokine and chemokine levels between T1D patients and brain-dead organ donors. ${ }^{45}$ 
Although cytokines are typically classified as pro- or anti-inflammatory, growing evidence suggests that the activity of a given cytokine may be influenced by the timing of response in disease course, its concentration and the co-existing cytokines in the local milieu. ${ }^{46,47}$

Indeed, our results demonstrate the importance of the bulk cytokine milieu. The fact that anti-inflammatory cytokines are typically expressed in conjunction with the pro-inflammatory cytokine TNF- $\alpha$ indicates that this might be a reactive response to counteract proinflammatory cytokine toxicity. The level of cytokine response possibly influences the remaining beta-cell reservoir in the first stages of disease as suggested by the observation that children with lower cytokine levels maintain higher C-peptide at onset and have longer remission phases.

Data on insulitis might explain the lower peripheral B-cell counts. In fact, B-cells represent one of the most abundant populations in the islets after CD8+ T-cells, ${ }^{48,49}$ being more frequent in the pancreatic inflammatory infiltrate of late $\mathrm{T} 1 \mathrm{D},{ }^{50}$ and inversely correlating with insulin-positivity. ${ }^{48}$ Recently, a higher prevalence of B-cells was associated with a more pronounced hyperimmune infiltration and a younger age at diagnosis. ${ }^{49}$ However, in our study, no age-correlation was found with B-cell counts.

Addressing study design, the purpose of using control samples was to compare children with and without diabetes. Control samples were collected simultaneously to $\mathrm{T} 1$, in different months along the 2 years of data collection. No further evaluations were performed in controls, as variations in their immune profile were not expected (controls did not have any immunologic or inflammatory disorder, there was no relevant seasonal clustering, and the study's time-span of circa 1 year, 398 days mean value of monitoring T1D patients, would not significantly account for age-related variations).

However, a larger sample size, including the evaluation of nonremitters, would strengthen the external validity in future studies, and further organ targeted and functional immune subset studies are required, to correlate the peripheral immune variations with the events occurring in the pancreas.

In conclusion, immune cell patterns and cytokine environment are relevant players in the natural course of the disease. The integrated longitudinal picture of T1D progression represents a basis for disease staging and appropriate patient stratification that will enable individualized approaches for resetting the immune system.

\section{ACKNOWLEDGEMENTS}

We thank Íris Caramalho from Instituto Gulbenkian de Ciência for her kind revision and comments, and Glória Nunes and Dagmar Lischke for technical support. This investigation was sponsored by Sanofi.

\section{ORCID}

Ana Laura Fitas (D) http://orcid.org/0000-0002-6667-7597

\section{REFERENCES}

1. Skyler JS, Bakris GL, Bonifacio E, et al. Differentiation of diabetes by pathophysiology, natural history, and prognosis. Diabetes. 2017;66: 241-255.
2. Patterson CC, Dahlquist GG, Gyurus E, Green A, Soltesz G. Incidence trends for childhood type 1 diabetes in Europe during 1989-2003 and predicted new cases 2005-20: a multicentre prospective registration study. Lancet. 2009;373:2027-2033.

3. Burton AR, Vincent E, Arnold PY, et al. On the pathogenicity of autoantigen-specific T-cell receptors. Diabetes. 2008;57:1321-1330.

4. Serreze DV, Fleming SA, Chapman HD, Richard SD, Leiter EH, Tisch RM. B lymphocytes are critical antigen-presenting cells for the initiation of $\mathrm{T}$ cell-mediated autoimmune diabetes in nonobese diabetic mice. J Immunol. 1998;161:3912-3918.

5. Noble JA, Valdes AM, Varney MD, et al. HLA class I and genetic susceptibility to type 1 diabetes: results from the type 1 diabetes genetics consortium. Diabetes. 2010;59:2972-2979.

6. Pugliese A. Insulitis in the pathogenesis of type 1 diabetes. Pediatr Diabetes. 2016;17(Suppl 22):31-36.

7. Battaglia M. Neutrophils and type 1 autoimmune diabetes. Curr Opin Hematol. 2014;21:8-15.

8. Aly H, Gottlieb P. The honeymoon phase: intersection of metabolism and immunology. Curr Opin Endocrinol Diabetes Obes. 2009;16: 286-292.

9. Chase HP, MacKenzie TA, Burdick J, et al. Redefining the clinical remission period in children with type 1 diabetes. Pediatr Diabetes. 2004;5:16-19.

10. Nagl K, Hermann JM, Plamper M, et al. Factors contributing to partial remission in type 1 diabetes: analysis based on the insulin dose-adjusted $\mathrm{HbA1c}$ in 3657 children and adolescents from Germany and Austria. Pediatr Diabetes. 2017;18:428-434.

11. Brown RJ, Rother KI. Effects of beta-cell rest on beta-cell function: a review of clinical and preclinical data. Pediatr Diabetes. 2008;9:14-22.

12. Jorns $A$, Arndt $T$, Meyer zu Vilsendorf $A$, et al. Islet infiltration, cytokine expression and beta cell death in the NOD mouse, BB rat, Komeda rat, LEW.1AR1-iddm rat and humans with type 1 diabetes. Diabetologia. 2014;57:512-521.

13. Itoh N, Hanafusa T, Miyazaki A, et al. Mononuclear cell infiltration and its relation to the expression of major histocompatibility complex antigens and adhesion molecules in pancreas biopsy specimens from newly diagnosed insulin-dependent diabetes mellitus patients. J Clin Invest. 1993;92:2313-2322.

14. Krogvold L, Edwin B, Buanes T, et al. Pancreatic biopsy by minimal tail resection in live adult patients at the onset of type 1 diabetes: experiences from the DiViD study. Diabetologia. 2014;57:841-843.

15. Bluestone JA, Herold K, Eisenbarth G. Genetics, pathogenesis and clinical interventions in type 1 diabetes. Nature. 2010;464: 1293-1300.

16. Kaas A, Pfleger C, Hansen L, et al. Association of adiponectin, interleukin (IL)-1ra, inducible protein 10, IL-6 and number of islet autoantibodies with progression patterns of type 1 diabetes the first year after diagnosis. Clin Exp Immunol. 2010;161:444-452.

17. Galgani M, Nugnes R, Bruzzese D, et al. Meta-immunological profiling of children with type 1 diabetes identifies new biomarkers to monitor disease progression. Diabetes. 2013;62:2481-2491.

18. Hamari S, Kirveskoski T, Glumoff V, et al. Analyses of regulatory CD4+ CD25+ FOXP3+ $T$ cells and observations from peripheral $T$ cell subpopulation markers during the development of type 1 diabetes in children. Scand J Immunol. 2016;83:279-287.

19. Diagnosis and classification of diabetes mellitus. Diabetes Care. 2014; 37(Suppl 1):S81-S90.

20. Mortensen HB, Hougaard P, Swift P, et al. New definition for the partial remission period in children and adolescents with type 1 diabetes. Diabetes Care. 2009;32:1384-1390.

21. Seddiki N, Santner-Nanan B, Martinson J, et al. Expression of interleukin (IL)-2 and IL-7 receptors discriminates between human regulatory and activated T cells. J Exp Med. 2006;203:1693-1700.

22. Liu W, Putnam AL, Xu-Yu Z, et al. CD127 expression inversely correlates with FoxP3 and suppressive function of human $\mathrm{CD} 4+\mathrm{T}$ reg cells. J Exp Med. 2006;203:1701-1711.

23. Valle A, Giamporcaro GM, Scavini M, et al. Reduction of circulating neutrophils precedes and accompanies type 1 diabetes. Diabetes. 2013;62:2072-2077.

24. Korsgren S, Molin Y, Salmela K, Lundgren T, Melhus A, Korsgren O. On the etiology of type 1 diabetes: a new animal model signifying a 
decisive role for bacteria eliciting an adverse innate immunity response. Am J Pathol. 2012;181:1735-1748.

25. Pelletier M, Maggi L, Micheletti A, et al. Evidence for a cross-talk between human neutrophils and Th17 cells. Blood. 2010;115:335-343.

26. Wojkowska DW, Szpakowski P, Ksiazek-Winiarek D, Leszczynski M, Glabinski A. Interactions between neutrophils, Th17 cells, and chemokines during the initiation of experimental model of multiple sclerosis. Mediators Inflamm. 2014;2014:590409.

27. Arif S, Moore F, Marks K, et al. Peripheral and islet interleukin-17 pathway activation characterizes human autoimmune diabetes and promotes cytokine-mediated beta-cell death. Diabetes. 2011;60: 2112-2119.

28. Ferraro A, Socci C, Stabilini A, et al. Expansion of Th17 cells and functional defects in T regulatory cells are key features of the pancreatic lymph nodes in patients with type 1 diabetes. Diabetes. 2011;60: $2903-2913$

29. Schwarzenberger P, La Russa V, Miller A, et al. IL-17 stimulates granulopoiesis in mice: use of an alternate, novel gene therapy-derived method for in vivo evaluation of cytokines. J Immunol. 1998;161: 6383-6389.

30. Marwaha AK, Crome SQ, Panagiotopoulos C, et al. Cutting edge: increased IL-17-secreting $\mathrm{T}$ cells in children with new-onset type 1 diabetes. J Immunol. 2010;185:3814-3818.

31. Honkanen J, Nieminen JK, Gao R, et al. IL-17 immunity in human type 1 diabetes. J Immunol. 2010;185:1959-1967.

32. Bradshaw EM, Raddassi K, Elyaman W, et al. Monocytes from patients with type 1 diabetes spontaneously secrete proinflammatory cytokines inducing Th17 cells. J Immunol. 2009;183:4432-4439.

33. Marek-Trzonkowska N, Mysliwiec $M$, Dobyszuk A, et al. Administration of CD4+CD25highCD127- regulatory $T$ cells preserves beta-cell function in type 1 diabetes in children. Diabetes Care. 2012;35:1817-1820.

34. Kukreja A, Cost G, Marker J, et al. Multiple immuno-regulatory defects in type-1 diabetes. J Clin Invest. 2002;109:131-140.

35. Ryba-Stanislawowska $M$, Skrzypkowska $M$, Mysliwiec $M$, Mysliwska J. Loss of the balance between CD4(+)Foxp3(+) regulatory T cells and CD4(+)IL17A(+) Th17 cells in patients with type 1 diabetes. Hum Immunol. 2013;74:701-707.

36. Marek N, Mysliwiec M, Raczynska K, Zorena K, Mysliwska J, Trzonkowski P. Increased spontaneous production of VEGF by CD4+ T cells in type 1 diabetes. Clin Immunol. 2010;137:261-270.

37. Ryba-Stanislawowska M, Skrzypkowska M, Mysliwska J, Mysliwiec M. The serum IL-6 profile and Treg/Th17 peripheral cell populations in patients with type 1 diabetes. Mediators Inflamm. 2013;2013:205284.

38. Jain R, Tartar DM, Gregg RK, et al. Innocuous IFNgamma induced by adjuvant-free antigen restores normoglycemia in NOD mice through inhibition of IL-17 production. J Exp Med. 2008;205:207-218.
39. Emamaullee JA, Davis J, Merani S, et al. Inhibition of Th17 cells regulates autoimmune diabetes in NOD mice. Diabetes. 2009;58: 1302-1311.

40. Rodacki M, Svoren B, Butty V, et al. Altered natural killer cells in type 1 diabetic patients. Diabetes. 2007;56:177-185.

41. Fraker C, Bayer AL. The expanding role of natural killer cells in type 1 diabetes and immunotherapy. Curr Diab Rep. 2016;16:109.

42. Atkinson MA, Eisenbarth GS. Type 1 diabetes: new perspectives on disease pathogenesis and treatment. Lancet. 2001;358:221-229.

43. Schloot NC, Hanifi-Moghaddam P, Aabenhus-Andersen N, et al. Association of immune mediators at diagnosis of type 1 diabetes with later clinical remission. Diabet Med. 2007;24:512-520.

44. Fatima N, Faisal SM, Zubair S, et al. Role of pro-inflammatory cytokines and biochemical markers in the pathogenesis of type 1 diabetes: correlation with age and glycemic condition in diabetic human subjects. PLoS One. 2016;11:e0161548.

45. Krogvold L, Wiberg A, Edwin B, et al. Insulitis and characterisation of infiltrating $T$ cells in surgical pancreatic tail resections from patients at onset of type 1 diabetes. Diabetologia. 2016;59:492-501.

46. Moudgil KD. Interplay among cytokines and T cell subsets in the progression and control of immune-mediated diseases. Cytokine. 2015;74:1-4.

47. Tisoncik JR, Korth MJ, Simmons CP, Farrar J, Martin TR, Katze MG. Into the eye of the cytokine storm. Microbiol Mol Biol Rev. 2012;76: 16-32.

48. Willcox A, Richardson SJ, Bone AJ, Foulis AK, Morgan NG. Analysis of islet inflammation in human type 1 diabetes. Clin Exp Immunol. 2009;155:173-181.

49. Arif $S$, Leete $P$, Nguyen $V$, et al. Blood and islet phenotypes indicate immunological heterogeneity in type 1 diabetes. Diabetes. 2014;63: 3835-3845.

50. In't Veld P. Insulitis in human type 1 diabetes: the quest for an elusive lesion. Islets. 2011;3:131-138.

\section{SUPPORTING INFORMATION}

Additional Supporting Information may be found online in the supporting information tab for this article.

How to cite this article: Fitas AL, Martins C, Borrego LM, et al. Immune cell and cytokine patterns in children with type 1 diabetes mellitus undergoing a remission phase: A longitudinal study. Pediatr Diabetes. 2018;19:963-971. https://doi. org/10.1111/pedi.12671 